\title{
Awareness during CPR on cardiac arrest due to aortic dissection
}

Lundsgaard RS, MD, Departement of Anaesthesiology, Nykøbing Falster Hospital and Department of Anaesthesiology Herlev Hospital, Denmark

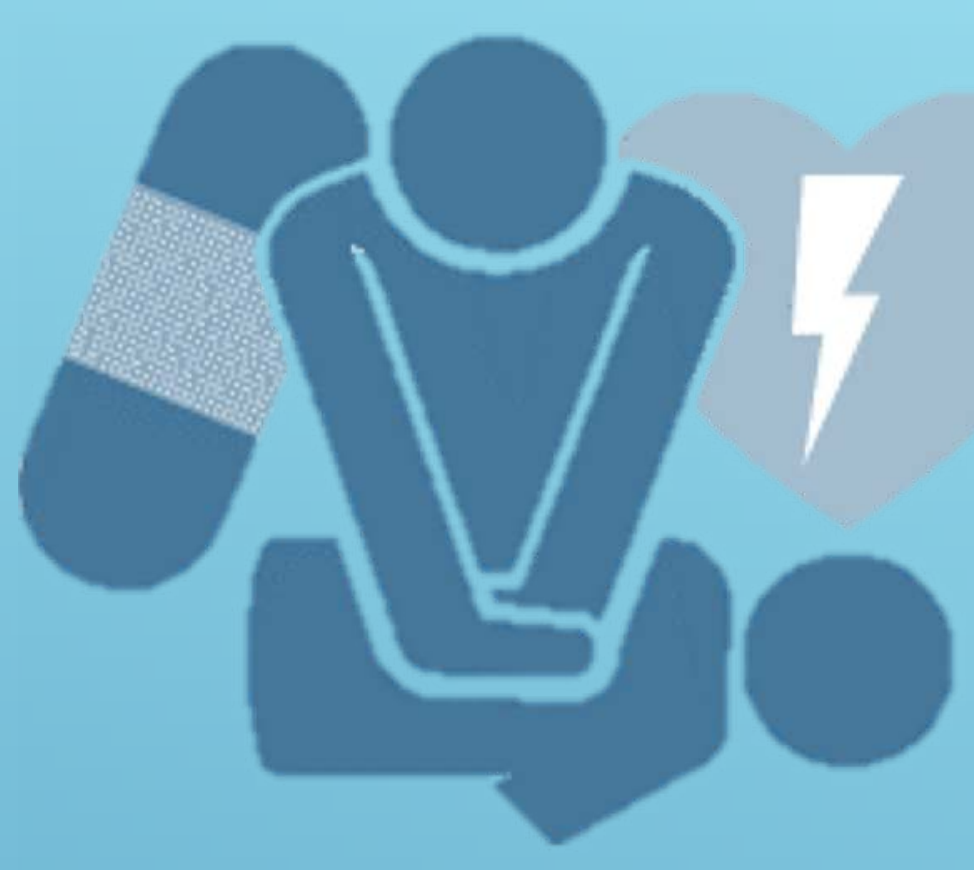

\section{Background}

Awareness during CPR is formerly reported in the literature but only in rare cases and it is still not clear whether these cases have anything in common.(1)

\section{Case report}

A 69-year old male is admitted to the hospital due to 3 days dyspeptic symptoms. The patient reports onset of dyspnea a few hours before arrival but has no other complains. During admission a short period of tachycardia $(250 / \mathrm{min})$ is followed by asystolic cardiac arrest and CPR is immediately initiated (chest compressions: 100/min and mask ventilation with $100 \%$ oxygen, ratio $30: 2$ ).

By the arrival of the cardio-resuscitation-team, the patient has a peripheral hemoglobin saturation of $100 \%$ and a high level of awareness with open eyes and movement of the head and both arms and legs. Re-evaluation of the hearts rhythm after 3 minutes still shows asystole.

A total of $20 \mathrm{mg}$ of Epinephrine is administered in boluses of $1 \mathrm{mg}$ every 3-5 minutes. By the 3rd hand-off for rhythm evaluation, the patient is orally intubated and there is still a high level of awareness as compressions continues. A total of 5 arterial gas samples are done during the 90 minute CPR, and despite a decrease in $\mathrm{pH}$ to 7,11 and lactate of 18 after 90 minutes all other markers remain within the normal range, and both the arterial and peripheral hemoglobin saturation remains $100 \%$.
The level of awareness does not decrease notably during the 90 minutes. Echocardiography is performed during hands-of periods by a cardiology consultant and a heart with no noticeable ultrasonic movement is seen. After one hour a projection visualizing the aortic arch raises suspicion of an aortic dissection. The patient is later shown by autopsy to have suffered a complete aortic dissection

\section{Discussion}

High level of awareness plus saturation and arterial gas almost within the normal range through 90 minutes indicate very effective chest compressions and peripheral and cerebral blood flow. Even though the patient had a poor prognosis (complete aortic dissection and refractory asystole), the termination of CPR raised ethical questions in the team as the patient was still conscious once the resuscitation was terminated after 90 minutes. Awareness during CPR also raises the question of proper sedation doing resuscitation, currently not part of the guideline.

\section{Learning points}

Pain relief and sedation during CPR Ethical aspects of termination of CPR

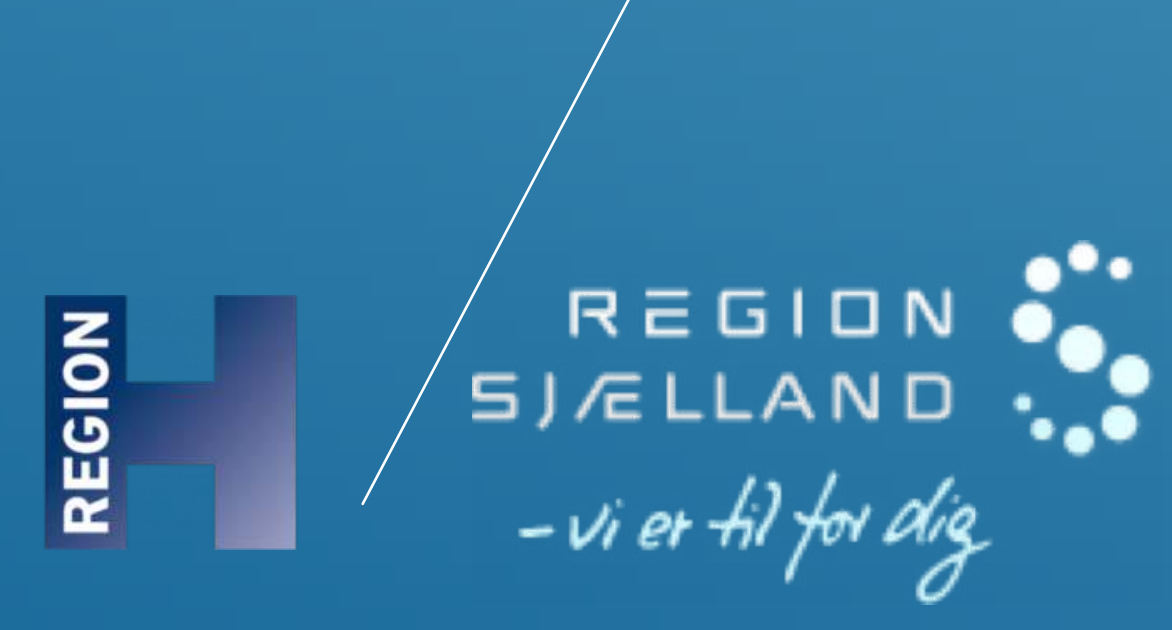

